\title{
C-reactive protein stimulates RAGE expression in human coronary artery endothelial cells in vitro via ROS generation and ERK/NF-KB activation
}

\author{
Yun ZHONG ${ }^{1, \#}$, Chuan-fang CHENG ${ }^{1, \#}$, Yi-zhi LUO ${ }^{1}$, Chao-wei TIAN ${ }^{1}$, Hui YANG ${ }^{1}$, Ben-rong LIU $^{1}$, Min-sheng CHEN $^{1}$, Yan-fang \\ $\mathrm{CHEN}^{1,2}$, Shi-ming $\mathrm{LIU}^{1, *}$ \\ ${ }^{1}$ Guangzhou Institute of Cardiovascular Disease, Second Affiliated Hospital of Guangzhou Medical University, Guangzhou 510260, \\ China; ${ }^{2}$ Department of Pharmacology \& Toxicology, Boonshoft School of Medicine, Wright State University, Dayton, OH 45324, USA
}

Aim: The receptor for advanced glycation end-products (RAGE) plays an important role in development of atherosclerosis, and C-reactive protein (CRP) has been found to stimulate its expression in endothelial cells. In this study we investigated how CRP regulated the expression of RAGE in human coronary artery endothelial cells (HCAECs).

Methods: HCAECs were treated in vitro with CRP $(50 \mu \mathrm{g} / \mathrm{mL})$ in combination with a variety of inhibitors. ROS generation was determined by immunocytochemistry and flow cytometry. The RAGE expression and phosphorylation of relevant signaling proteins were measured using Western blot analyses.

Results: CRP stimulated the expression of RAGE in the cells, accompanied by markedly increased ROS generation, phosphorylation of ERK1/2 and NF-KB p65, as well as translocation of NF-kB p65 to the nuclei. CRP also stimulated phosphorylation of JNK and p38 MAPK. Pretreatment of the cells with the ROS scavenger N-acetyl-L-cysteine, ERK inhibitor PD98059 or NF-kB inhibitor PDTC blocked CRP-stimulated RAGE expression, but pretreatment with the NADPH oxidase inhibitor DPI, JNK inhibitor SP600125 or p38 MAPK inhibitor SB203580 did not significantly alter CRP-stimulated RAGE expression.

Conclusion: CRP stimulates RAGE expression in HCAECs in vitro via ROS generation and activation of the ERK/NF-KB signaling pathway.

Keywords: C-reactive protein; advanced glycosylation end products; reactive oxygen species; NF-kappa B; endothelial cells; p38 mitogen-activated protein kinases; atherosclerosis; extracellular signal-regulated MAP kinases

Acta Pharmacologica Sinica (2015) 36: 440-447; doi: 10.1038/aps.2014.163; published online 23 Mar 2015

\section{Introduction}

Plasma C-reactive protein (CRP) is known as a prototypic marker of inflammation ${ }^{[1]}$. Increasing evidence suggests that CRP is a pro-inflammatory cytokine that contributes to the initiation and progression of atherosclerosis by promoting endothelial activation and macrophage recruitment ${ }^{[2,3]}$. CRP mostly binds to cell membrane IgG Fcy receptors to elicit proatherogenic changes, such as the expression of macrophage chemoattractant protein 1 (MCP-1) and vascular cell adhesion molecule 1 (VCAM-1) ${ }^{[4,5]}$.

Reactive oxygen species (ROS) are linked to the overexpression of pro-inflammatory mediators that play a critical role in endothelial activation and atherosclerosis ${ }^{[6-8]}$. As one of the

\footnotetext{
\#These authors contributed equally to this article.

* To whom correspondence should be addressed.

E-mail gzliushiming@126.com

Received 2014-10-29 Accepted 2014-12-09
}

pro-inflammatory mediators, CRP upregulates ROS production in endothelial cells, platelets, monocytes, and vascular smooth muscle cells via specific $\mathrm{Fc}_{\mathrm{C}}$ receptors ${ }^{[9,10]}$. It has been reported that $\mathrm{CRP}$ upregulates $\mathrm{MCP}-1$ expression in monocytes via nicotinamide adenine dinucleotide phosphate-oxidase (NADPH oxidase)/ROS mediated nuclear factor-kB (NFКB) and p38 mitogen-activated protein kinases (p38 MAPK) activation $^{[11]}$. CRP has been shown to promote macrophage uptake of oxidized low-density lipoprotein (ox-LDL) via the ROS/NF-KB pathway ${ }^{[9]}$. In human umbilical vein endothelial cells, CRP up-regulates the expression and activity of tissue factor pathway via NF- $\mathrm{KB}$ and extracellular regulated protein kinases1/2 (ERK1/2) pathway ${ }^{[12]}$. These studies indicate that ROS, ERK1/2, p38 MAPK and NF-кB link together to mediate CRP-induced pro-atherosclerotic activation.

Advanced glycation end products (AGEs) and their receptor RAGE have been shown to play an important role in endothelial activation and inflammation ${ }^{[13]}$. The deletion of 
bone marrow-derived RAGE inhibits atherosclerotic plaque progression $^{[14]}$, and blockade of RAGE signaling almost completely inhibits the development of diabetes-associated atherosclerosis ${ }^{[15]}$. Interestingly, CRP has been shown to up-regulate the expression of RAGE via ERK, p38 and JNK pathways in the human monocytic THP-1 cell line (THP-1 cells) ${ }^{[16]}$. We have demonstrated that CRP upregulates the expression of RAGE and that silencing RAGE gene prevents CRP-induced MCP-1 secretion in human saphenous vein endothelial cells ${ }^{[17]}$. These observations suggest that CRP-induced RAGE expression participates in endothelial activation and atherosclerosis. However, the underlying signaling pathway by which CRPinduced RAGE expression exerts its effects has not been fully elucidated.

The aim of the present study was to investigate the underlying signaling pathways involved in CRP-induced RAGE expression in human endothelial cells.

\section{Materials and methods Materials}

Purified human recombinant native CRP was obtained from Trichem Resources Inc (West Chester, PA, USA). NF-кB inhibitor pyrrolidine dithiocarbamate (PDTC), ERK inhibitor (PD98059), p38 MAPK inhibitor (SB203580) and JNK inhibitor (SP600125) were purchased from Calbiochem (San Diego, CA, USA). The antioxidant $N$-acetyl-L-cysteine (NAC) and the NADPH oxidase inhibitor diphenyleneiodonium (DPI) were purchased from Sigma (St Louis, MO, USA).

\section{Cell culture}

Primary human coronary artery endothelial cells (HCAECs) were obtained from Cell Applications Inc (San Diego, CA, USA) and grown in endothelial cell basal medium-2 (EBM2) supplemented with EGM-2MV single-use aliquots, as described by the supplier (Lonza Inc, Lonza CA, USA). Cells from passages 3-6 were used for further experiments.

\section{Reactive oxygen species (ROS) detection}

To detect ROS in the living cells, an ROS detection kit was used (Image-iT LIVE Green Reactive Oxygen Species Detection Kit, Molecular Probes, Invitrogen). The kit was applied as described in the manufacturer's protocol. Briefly, HCAECs were seeded into 4-well cell culture chamber slides as previously described. Following incubation with different agents, intracellular and unspecific ROS were marked with $2^{\prime}, 7^{\prime}$-dichlorodihydrofluorescein diacetate $\left(\mathrm{H}_{2} \mathrm{DCFDA}\right.$, a general ROS indicator), and the cell nuclei with Hoechst 33342 solution. The cells were then washed 3 times with HBSS (Gibco, CA, USA). The cell culture samples were examined with a fluorescent microscope (Zeiss Axioplan; 10×40) equipped with an FITC and DAPI filter. To quantify ROS production, total ROS detection kit for flow cytometry (Enzo Life Sciences) was used as described in the manufacturer's protocol. Cells were analyzed using a Beckman FC 500 MPL flow cytometer with CXP LMD Acquisition \& Analysis software. The fluorescence intensity of 20000 cells for each sample was quantified.

\section{Confocal microscopy for NF-kB p65 localization}

HCAECs $\left(0.5 \times 10^{4}\right.$ cells/well) were seeded onto 4 -well cell culture chamber slides (BD Falcon) and cultured in EBM-2 supplemented with 10\% FBS (Gibco, CA, USA). After overnight growth, cells at $80 \%$ confluence were serum-starved for $24 \mathrm{~h}$ and incubated in serum-free EBM-2. Then, cells were rinsed twice with PBS and fixed with $3.7 \%$ formaldehyde for 15 min at room temperature, washed in PBS (3 times for $5 \mathrm{~min}$ ), permeabilized with $0.1 \%$ Triton X-100 in PBS for $15 \mathrm{~min}$, and blocked in Image-iT FX Signal Enhancer (Invitrogen, Carlsbad, CA, USA) for one hour at room temperature. Cells were then incubated with primary antibody of monoclonal mouse antihuman p65 (1:50, CST Inc, Beverly, MA), followed by incubation with secondary antibody of Alexa Fluor 488-conjugated goat anti-mouse IgG (1:1000, Invitrogen) for $30 \mathrm{~min}$ at room temperature, and actin filaments were labeled with Alexa Fluor 555 phalloidin (Invitrogen, USA) for $20 \mathrm{~min}$. After two washes with PBS, samples were mounted with ProLong Gold antifade reagent with DAPI (Invitrogen) and assessed twentyfour hours later using a laser-scanning confocal microscopy (×63 PlanApo 1.4 aperture objective; Zeiss LSM 710). Triple staining (Merge) for actin filaments or p65 and DAPI was performed to demonstrate the nucleus co-localization of NF- $\mathrm{KB}$ p65 subunit.

\section{Phospho-NF-kB p65 ELISA}

To evaluate the NF- $\mathrm{kB}$ activation, the endogenous phosphoNF-кB p65 was detected by phospho-NF-кB p65 (Ser536) Sandwich ELISA Kit (CST) according to the manufacturer's protocol. In brief, a phospho-NF-kB p65 mouse mAb was coated onto the microplate. After incubation with $100 \mu \mathrm{g}$ cell lysate from each cell preparation, phospho-NF-кB p65 proteins were captured by the coated antibody. Following extensive washing, NF- $\mathrm{kB}$ p65 rabbit $\mathrm{mAb}$ was added to detect the captured phospho-NF-kB p65 protein. Anti-rabbit IgG, HRPlinked antibody was then used to recognize the bound detection antibody. HRP substrate, TMB, was added to develop color. The absorbance at $450 \mathrm{~nm}$ was measured using a Tecan Infinite 200 microplate reader, and phospho-NF-kB p65 levels were normalized to the levels of total protein.

\section{Western blot analysis}

HCAECs lysates were fractionated through a 4\% stacking and $10 \%$ resolving SDS-PAGE gel, and the fractionated proteins were transferred to PVDF membranes. Membranes were blocked for one hour at room temperature with Odyssey Blocking Buffer. Western blots were developed using antiRAGE (1:100, Santa Cruz, CA), anti-beta-actin (1:200, Thermo Scientific, Fremont, CA), anti-ERK1/2 (1:2000, CST), anti-phospho-ERK1/ 2 (1:2000, CST), anti-phospho-p38 (1:2000, CST), and anti-phospho-JNK (1:1000, CST). Anti-mouse and antirabbit secondary antibodies conjugated to IRDye680 (1:10000, Li-Cor, NE) and IRDye800 (1:10000, Li-Cor), respectively, were used to probe primary antibodies. Protein bands were 
detected and quantified by the Odyssey system (Li-Cor).

\section{Statistical analyses}

All of the values are presented as the mean \pm SEM. Comparisons between multiple treatments groups were carried out with one-way ANOVA followed by a Tukey's test. Comparisons within 2 treatment groups were analyzed by Student's $t$-test. Differences were considered significant at $P<0.05$.

\section{Results}

CRP-induced ERK phosphorylation and RAGE expression were markedly reduced by ROS other than NADPH oxidase inhibitor

To clarify the involvement of ROS in CRP-induced RAGE expression, ROS inhibitor $N$-acetyl-L-cysteine (NAC) and NADPH oxidase inhibitor diphenyleneiodonium (DPI) were used. As shown in Figure 1A, ROS production was significantly increased by CRP $(50 \mu \mathrm{g} / \mathrm{mL})$. NAC $(30 \mathrm{mmol} / \mathrm{L})$ attenuated CRP-induced ROS. However, DPI $(10 \mu \mathrm{mol} / \mathrm{L})$ showed an insignificant inhibitory effect on CRP-induced ROS. The effects of the two ROS quenchers on CRP-induced ROS generation were further studied by flow-cytometry (Figure 1B). Moreover, in order to investigate the effect of ROS on CRP-activated ERK pathway, the phosphorylation of ERK 1/2 was examined after NAC and DPI pretreatment. Compared with the vehicle, NAC inhibited CRP-induced ERK $1 / 2$ phosphorylation (Figure 1C) and decreased CRP-induced RAGE protein level (Figure 1D).

CRP-induced NF-KB activation and RAGE expression were significantly attenuated by ERK inhibitor

To verify the role of ERK pathway in CRP-induced RAGE expression, HCAECs were pretreated with ERK1/2 inhibitor (PD98059, $50 \mu \mathrm{mol} / \mathrm{L})$ for one hour followed by CRP (50 $\mu \mathrm{g} / \mathrm{mL}$ ) treatment for three hours (for detecting ERK1/2 phosphorylation) or twelve hours (for detecting RAGE \& $\beta$-actin expression ${ }^{[12]}$. As shown in Figure $2 \mathrm{~A}$ and $2 \mathrm{~B}, \mathrm{CRP}$ increased the phosphorylation of ERK 1/2 in HCAECs, but this effect was prevented by PD98059. After treatment with CRP for twelve hours, the effect of CRP-induced RAGE expression was blocked by PD98059 $(P<0.01)$. To determine whether NF-kB is down-stream of CRP-induced ERK $1 / 2$ phosphorylation, the subcellular localization of NF-kB p65 subunits was studied. As shown in Figure 2C, CRP stimulated a translocation of NF-kB p65 subunits, which was blocked by pretreating the cells with PD98059. The detection of phosphorylated NF-kB p65 levels also confirmed the inhibitory effects of PD98059 (Figure 2D). These data suggest that CRP increases RAGE expression through activation of the ERK1/2-NF-KB pathway.

CRP-induced RAGE expression was dramatically suppressed by NF-KB inhibitor

To examine whether NF-KB signaling pathway is involved in CRP-induced RAGE expression, HCAECs were pretreated with the NF-kB inhibitor (PDTC, $100 \mu \mathrm{mol} / \mathrm{L}$ ) for one hour followed by CRP $(50 \mu \mathrm{g} / \mathrm{mL})$ treatment for six hours (for detecting NF-kB phosphorylation and translocation) or twelve hours (for detecting RAGE \& $\beta$-actin expression) ${ }^{[12,18]}$. NF-кB activation was demonstrated by detecting nuclear translocation and the phosphorylation of NF-kB p65. Treating HCAECs with $\mathrm{CRP}$ resulted in sustained nuclear accumulation of NF-kB p65 subunit, which was inhibited by pretreating cells with PDTC (Figure 3A). Concomitantly with the nuclear localization of p65, phospho-p65 level was significantly increased in CRPtreated cells $(P<0.05)$ and decreased both in PDTC-treated cells in the presence or absence of CRP (Figure 3B). The data were confirmed by Western blot, which showed significantly increased of RAGE protein expression after CRP treatment. Moreover, CRP-induced RAGE expression was inhibited by PDTC pretreatment (Figure 3C). These results suggest that CRP induces RAGE expression via NF-KB activation.

\section{CRP-induced RAGE protein expression was not affected by p38} MAPK or JNK inhibitor

To investigate whether p38 MAPK and JNK are involved in CRP-induced RAGE expression, specific p38 MAPK inhibitor (SB203580) and JNK inhibitor (SP600125) were used in the present study. SB203580 and SP600125 inhibited CRP-induced p38 and JNK phosphorylation, respectively. SB203580 had no inhibitory effect on JNK phosphorylation, but SP600125 significantly increased the phosphorylation of p38 MAPK (Figure 4A). However, both inhibitors had no effect in CRP-induced RAGE expression (Figure 4B).

\section{Discussion}

CRP has been proposed to play a pivotal role in vascular inflammation and dysfunction ${ }^{[19]}$. Meanwhile, a large body of evidence suggests that RAGE plays an important role in the development of atherosclerosis ${ }^{[20]}$. Our previous work was the first to demonstrate that CRP increases the level of MCP-1 in human saphenous vein endothelial cells via the upregulation of RAGE expression, suggesting RAGE could be the key protein in CRP-mediated pro-inflammatory response ${ }^{[17]}$. In this study, we further investigated the underlying intracellular signals involved in CRP-induced RAGE upregulation in HCAECs. Our data showed that CRP induces RAGE expression in HCAECs and is accompanied by ROS overproduction, ERK1/2 phosphorylation and NF-KB activation. Interestingly, CRP-induced ROS production, ERK1/2 phosphorylation and RAGE expression were reduced by ROS inhibitor. Meanwhile, CRP-induced NF-KB activation and RAGE expression were decreased by either ERK or NF-KB inhibitors. However, CRPinduced RAGE expression was not affected by the inhibitor of NADPH oxidase, p38 MAPK or JNK.

It has been suggested that inflammation following exposure to CRP is highly dependent on oxidative stress, the mechanism by which CRP induces pro-inflammatory effects via the overproduction of several pro-inflammatory mediators such as MCP-1, ICAM, and VCAM- $1^{[9]}$. As we expected, our data showed that CRP increases ROS production in HCAECs. This is consistent with an observation of CRP-induced ROS production in endothelial progenitor cells ${ }^{[21]}$. Given that mitochondria and NADPH oxidases are the main cellular sources of 
A
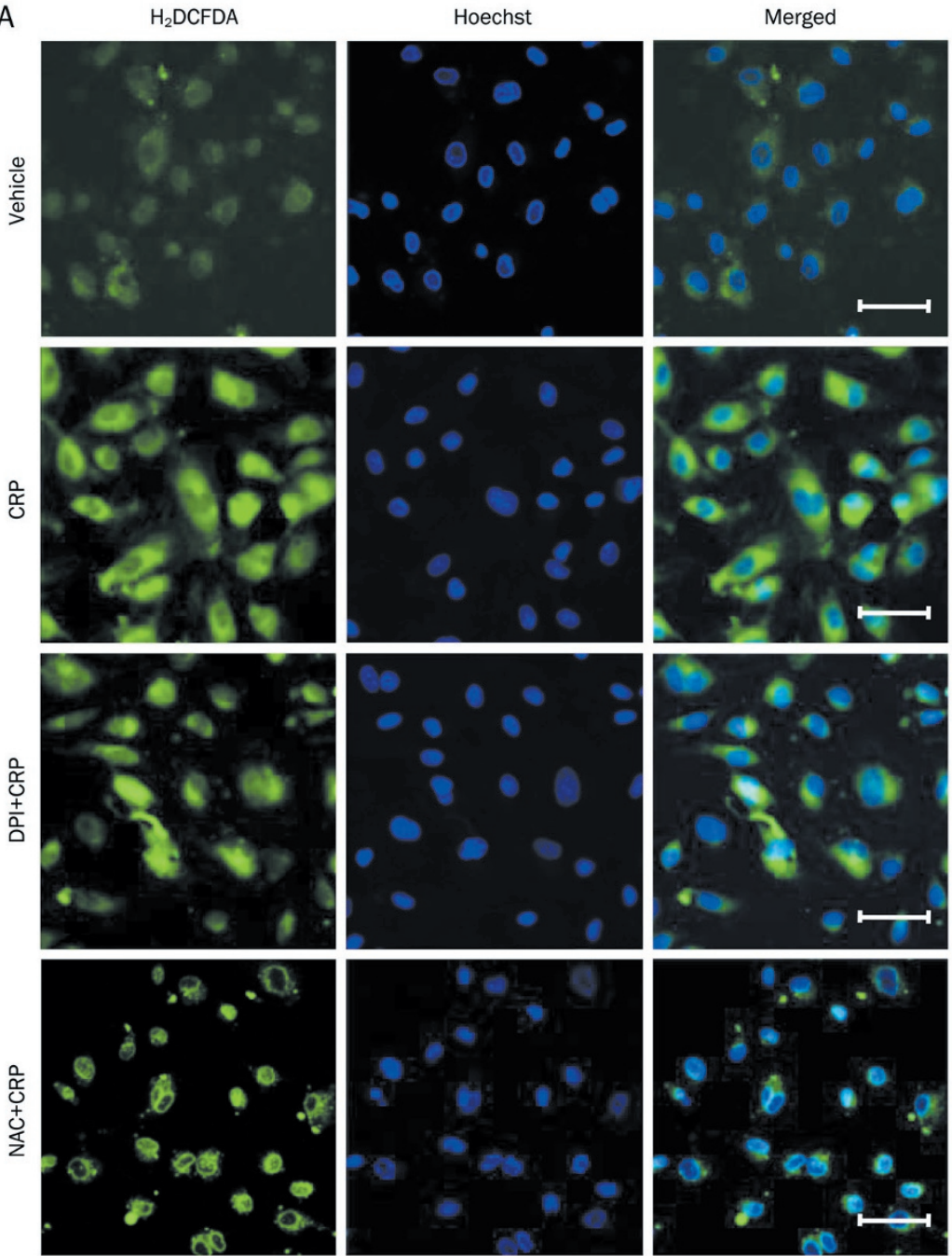

C
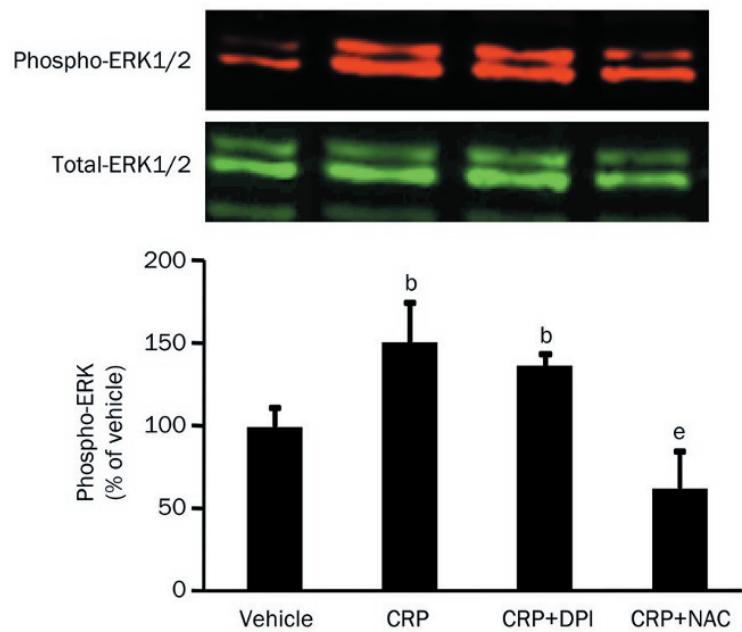

B1

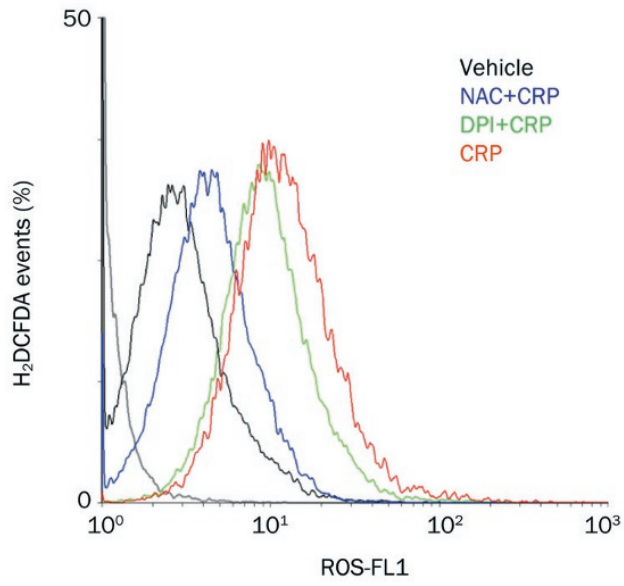

B2

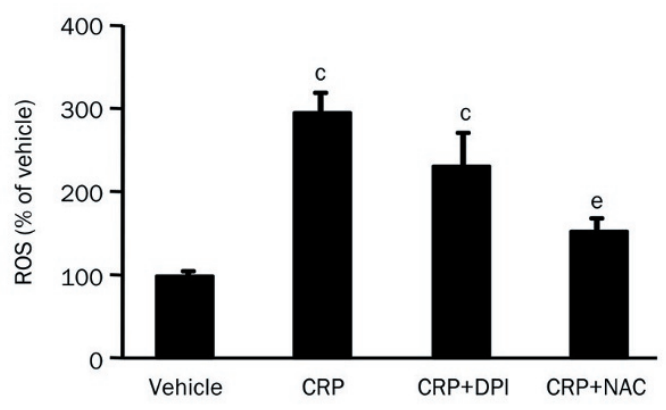

Figure 1. Effects of ROS or NADPH inhibitor on CRP-induced ROS production, RAGE expression and ERK phosphorylation in HCAECs. (A) Representative 2',7'-dichlorodihydrofluorescein diacetate $\left(\mathrm{H}_{2} \mathrm{DCFDA}\right.$, a general ROS indicator) under a fluorescent microscope (Scale bar: $\left.50 \mu \mathrm{m}\right)$. (B1) Representative flow cytometer analyses of ROS production in HCAECs treated with vehicle, CRP, diphenyleneiodonium (DPI, a NADPH oxidase inhibitor) with CRP or $\mathrm{N}$-acetyl-L-cysteine (NAC, a general ROS inhibitor) with CRP; (B2) Summarized fluorescence intensity of 20000 cells for each group. (C) Western blot analysis of phospho-ERK1/2 expression in HCAECs. (D) Western blot analysis of RAGE expression in HCAECs. Data are represented as the mean \pm SEM (n=3-6). ${ }^{\mathrm{b}} P<0.05,{ }^{\mathrm{c}} P<0.01$ vs vehicle. ${ }^{\mathrm{e}} P<0.05$ vs CRP. 
A

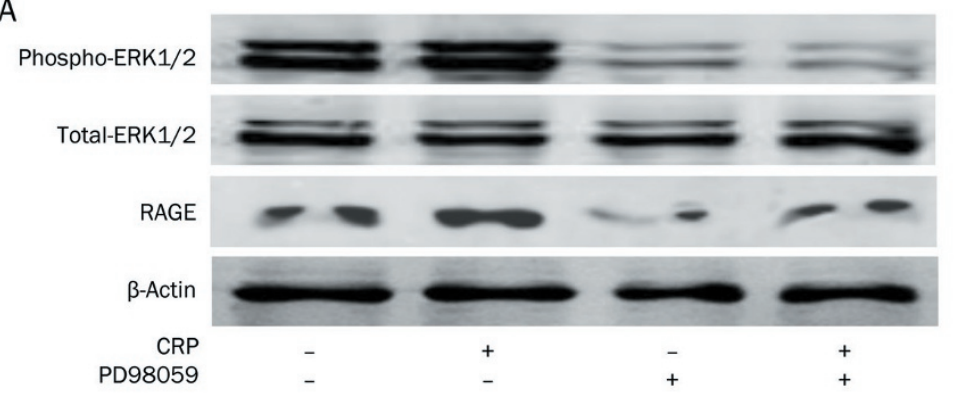

C
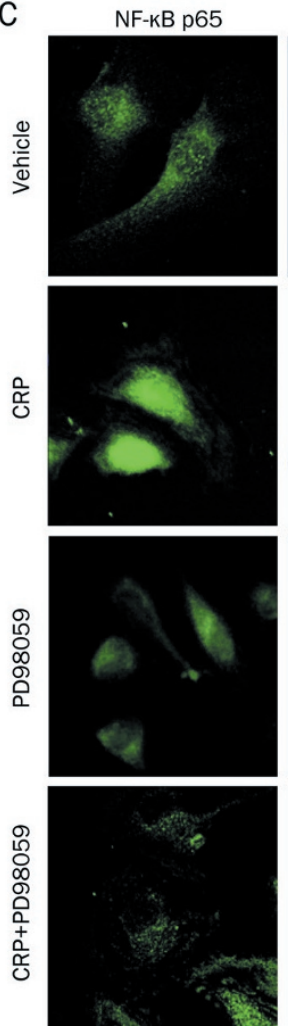

DAPI
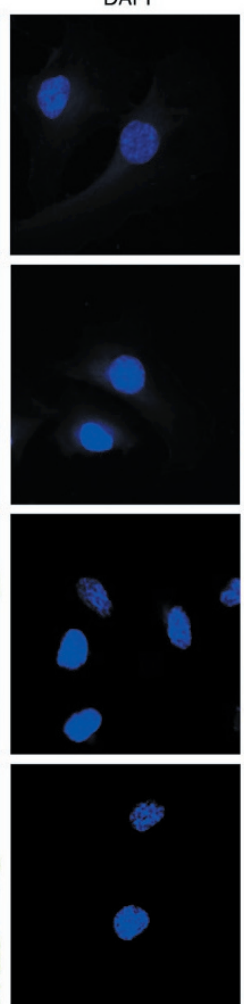

Phalloidin
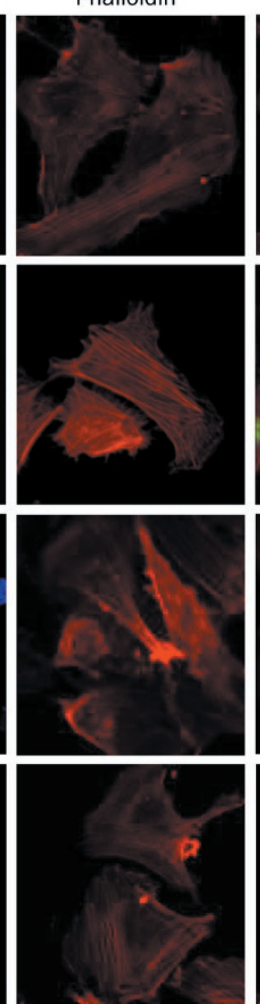

Merged
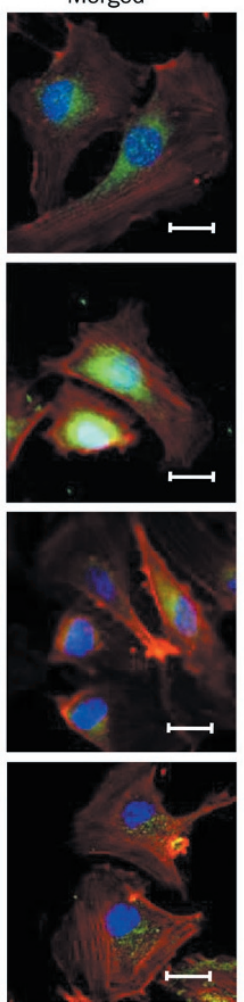

B

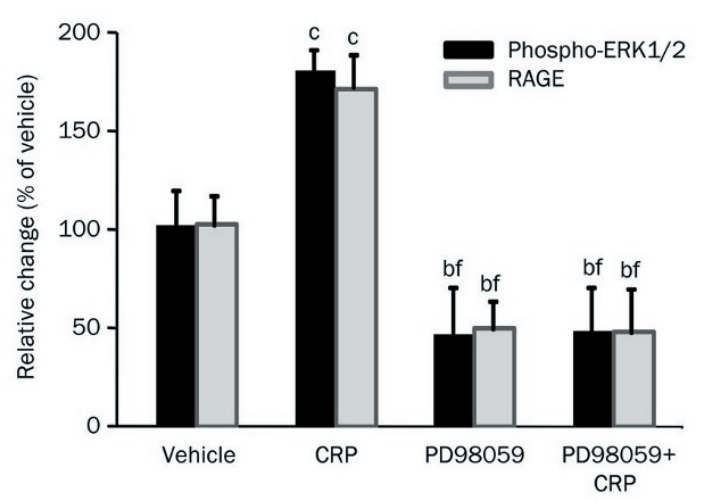

D

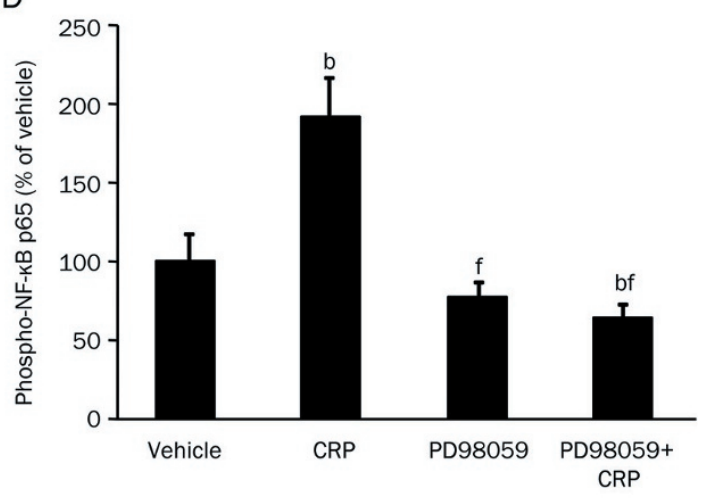

Figure 2. Effects of ERK1/2 inhibitor on CRP-induced ERK1/2 phosphorylation, NF-kB p65 phosphorylation and RAGE expression in HCAECs. (A and B) Western blot analysis of ERK1/2 phosphorylation and RAGE expression in HCAECs treated with vehicle, CRP, PD98059 (ERK1/2 inhibitor) or PD98059 with CRP. (C) Confocal microscopy analysis of NF-KB p65 (green) localization in HCAECs. The nuclei were stained with DAPI (blue), and the cell architecture was illustrated by Alexa Fluor 555-labeled phalloidin (red); scale bar: $20 \mu \mathrm{m}$. (D) ELISA analysis of phosphorylated NF-KB p65 in HCAECs. Data are represented as the mean \pm SEM $(n=3-6) .{ }^{b} P<0.05,{ }^{c} P<0.01$ vs vehicle. ${ }^{e} P<0.05,{ }^{\text {f }} P<0.01$ vs CRP.

ROS, we then sought to identify the source of CRP-increased ROS production. We found that the general ROS inhibitor NAC, but not the NADPH oxidase specific inhibitor DPI, decreased CRP-induced ROS generation, suggesting that mitochondria are the main source of CRP-induced ROS production in HCAECs. It should be noted that previous studies in porcine coronary endothelial cells and THP-1 cells suggested that CRP upregulates ROS production via activating the NADPH oxidase $^{[22,23]}$. These conflicting findings may be due to differences in cell type and species.

Importantly, we found that CRP-induced ROS generation was accompanied by ERK $1 / 2$ phosphorylation, NF-KB phosphorylation and RAGE production. Moreover, ROS inhibitor
NAC decreased ERK1/2 phosphorylation and RAGE expression, suggesting ROS is upstream of ERK1/2 and RAGE. This finding is in agreement with previous studies showing that CRP activates ERK1/2 in endothelial cells ${ }^{[12]}$, macrophages ${ }^{[24]}$ and cardiomyocytes ${ }^{[25]}$. Meanwhile, we used a selective ERK1/2 inhibitor PD98059 to determine whether ERK1/2 is involved in CRP-induced RAGE expression. We found that ERK1/2 inhibition attenuated CRP-induced RAGE expression in HCAECs, suggesting that ERK1/2 is upstream of RAGE expression. This is supported by a previous study in THP-1 cells showing that ERK1/2 is involved in CRP-induced RAGE expression $^{[16]}$. Moreover, we found that ERK1/2 inhibition attenuated CRP-induced NF- $\mathrm{KB}$ activation, indicating that NF- 
A
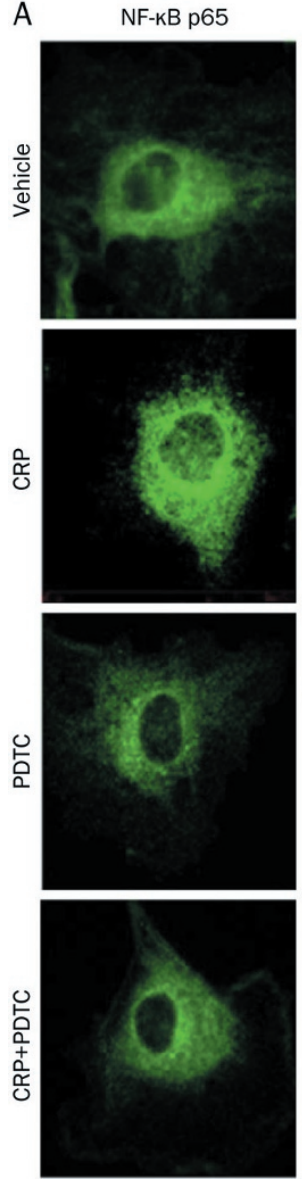

DAPI
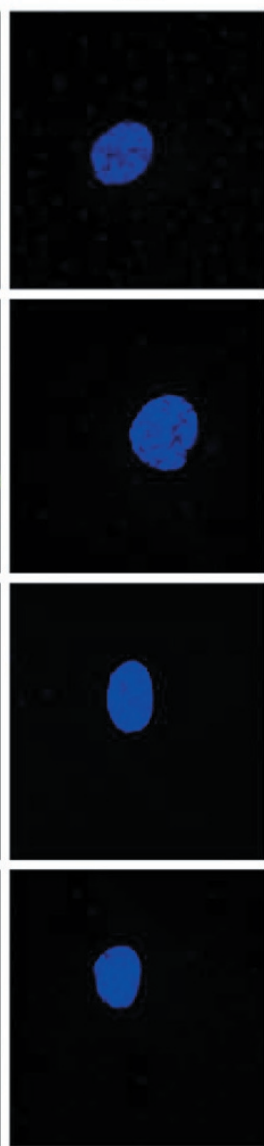

Phalloidin
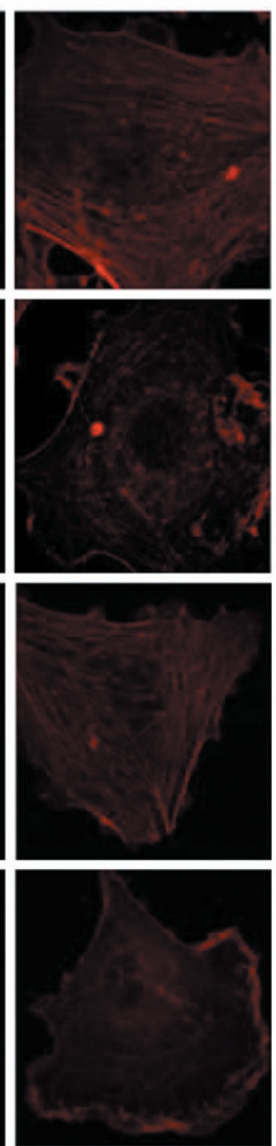

Merged
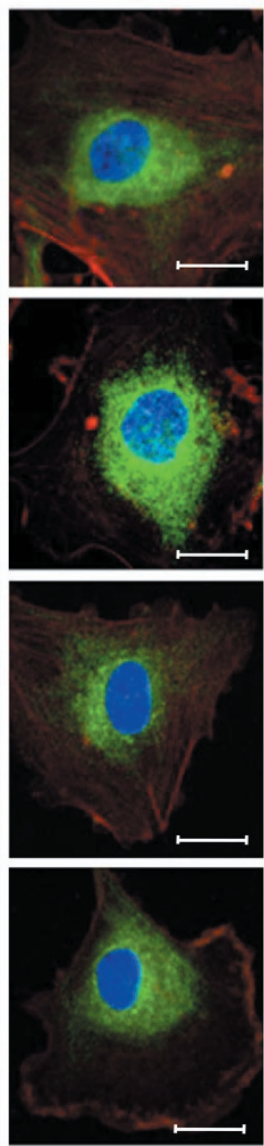

B

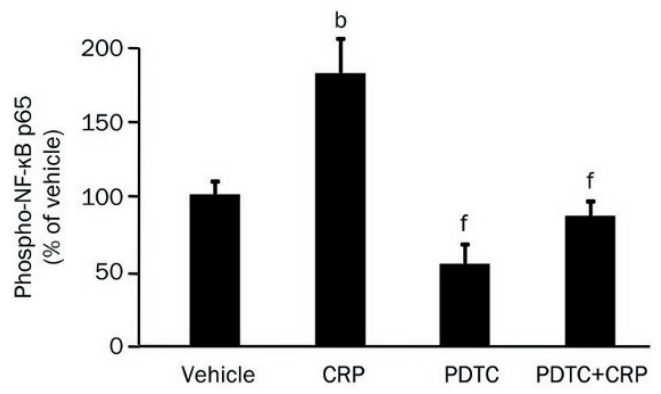

C
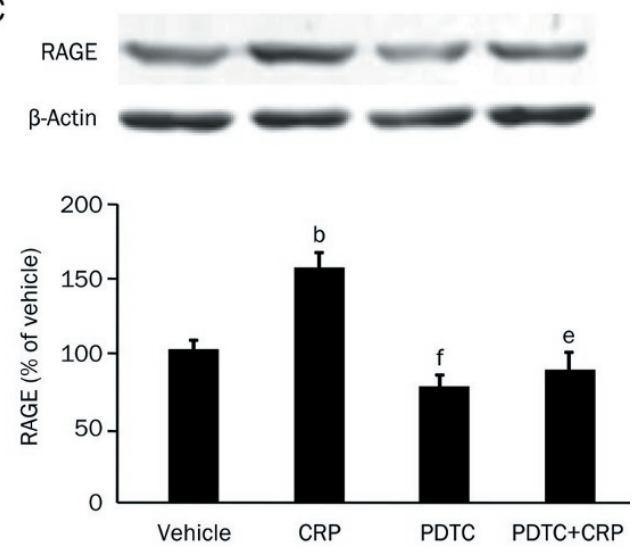

Figure 3. Effects of NF-kB inhibitor on CRP-induced NF-kB p65 location, phosphorylation and RAGE expression in HCAECs. (A) Confocal microscopy analysis of NF-KB p65 (green) localization in HCAECs treated with vehicle, CRP, PDTC, or PDTC with CRP. The nuclei were stained with DAPI (blue), and the cell architecture was illustrated by Alexa Fluor 555-labeled phalloidin (red); scale bar: $20 \mu \mathrm{m}$. (B) ELISA analysis of phosphorylated NF-KB p65 in HCAECs. (C) Western blot analysis of RAGE expression in HCAECs. Data are represented as the mean \pm SEM $(n=3-6)$. ${ }^{\mathrm{b}} P<0.05$ vs vehicle. ${ }^{\mathrm{e}} P<0.05$, ${ }^{\mathrm{f}} P<0.01$ vs CRP.

$\kappa \mathrm{B}$ is a downstream molecule of ERK1/2.

$\mathrm{NF}-\mathrm{kB}$ mediated vascular inflammation plays a critical role in the initiation and progression of atherosclerosis ${ }^{[26]}$. In the vascular endothelium, NF- $\mathrm{KB}$ activation induces the expression of proinflammatory genes, including those encoding adhesion molecules, cytokines, and chemoattractant proteins that collectively play critical roles in the initiation and progression of atherosclerosis ${ }^{[27]}$. Moreover, endothelial-specific NF- $\mathrm{kB}$ inhibition is sufficient to confer atheroprotection ${ }^{[28]}$. Previous studies have shown that CRP induces the expression of VCAM-1, ICAM-1 and MCP-1 in endothelial cells via NF- $\mathrm{kB}$ activation ${ }^{[29,30]}$. We investigated whether NF- $\mathrm{KB}$ is involved in CRP-induced RAGE expression by using the NF- $\mathrm{kB}$ inhibitor PDTC. As we expected, CRP increased the phosphorylation and nuclear translocation of NF-kB p65 and RAGE expression. Importantly, we found that the inhibition of NF- $\mathrm{kB}$ suppressed CRP-induced RAGE expression, suggesting that NF- $\mathrm{KB}$ is the upstream mediator of CRP-induced RAGE expression.

JNK and p38 MAPK are also key molecules of the MAPK signaling family. To investigate whether JNK and p38 MAPK are involved in CRP-induced RAGE expression, their respective inhibitors were used for the study. We found that CRPinduced RAGE expression was not affected by the inhibition of JNK or p38 MAPK although CRP increased their phosphorylation, indicating that CRP-induced RAGE expression is independent of p38 MAPK or JNK. Interestingly, our data also showed that JNK inhibitor significantly increased the phosphorylation of p38 MAPK. This finding is supported by a previous study in a pancreatic beta-cell line indicating that inhibition of JNK activates cAMP response element binding protein partially via the p38 MAPK pathway ${ }^{[31]}$. Altogether, our observations suggest there is a reciprocal regulation of p38 MAPK and JNK in the HCAECs. Further research is needed to understand the mechanisms underlying the regulatory effect of JNK on p38 MAPK.

In summary, our findings provide evidence for the involvement of ROS/ERK/NF-kB signaling pathways in CRPinduced RAGE expression (Figure 5). This molecular mechanism could be important in the pathogenesis of endothelial inflammation, especially among patients with diabetes and, 
A
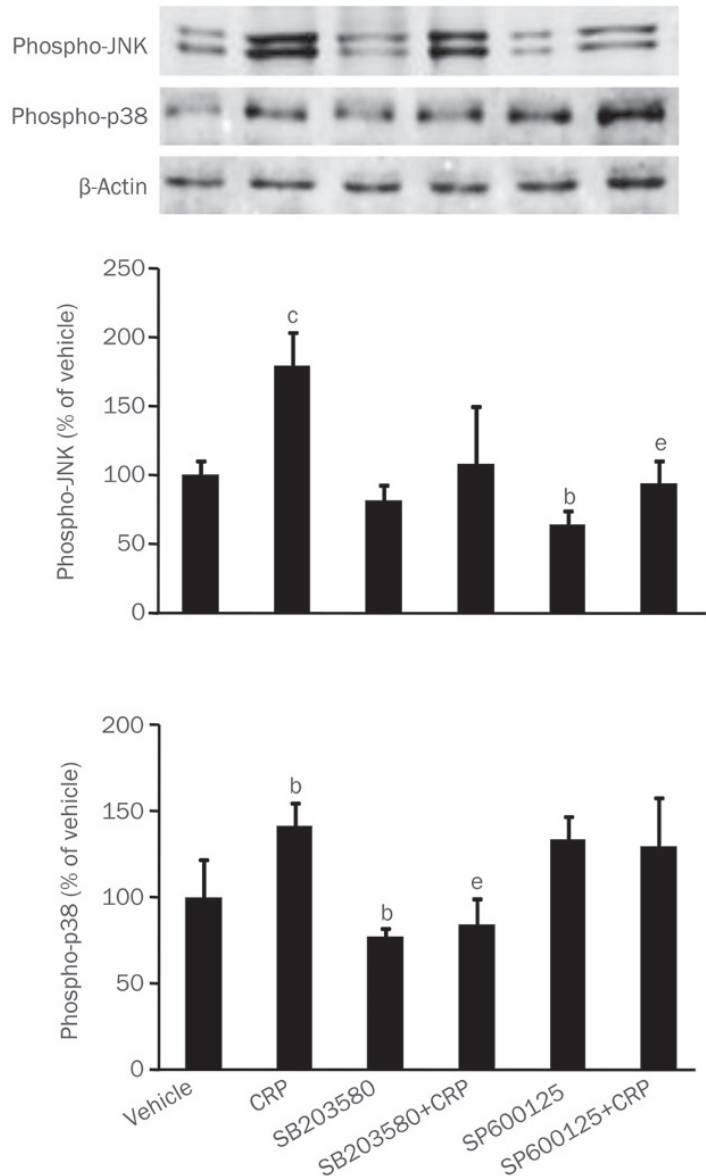

B
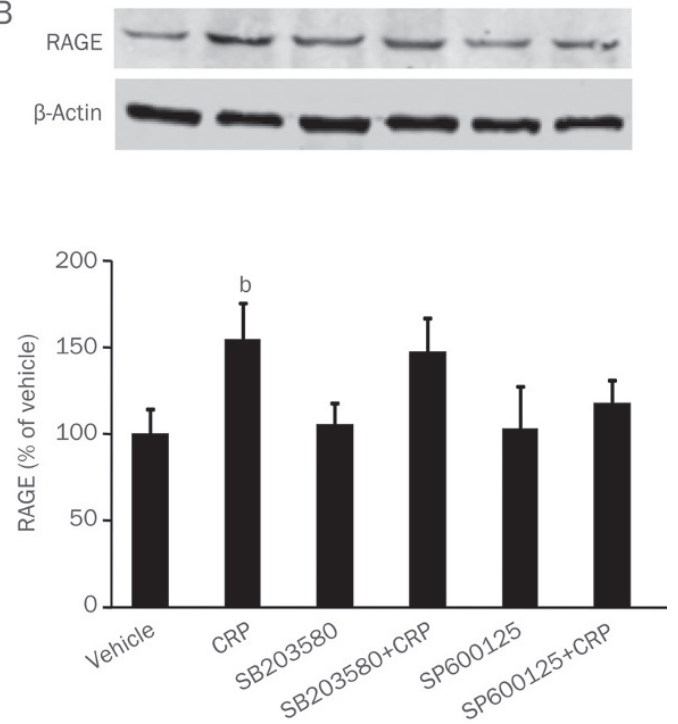

Figure 4. Effects of JNK or p38 MAPK inhibitor on CRP-induced JNK phosphorylation, p38 MAPK phosphorylation, NF-KB p65 phosphorylation and RAGE expression in HCAECs. (A) Western blot analysis of JNK phosphorylation and p38 MAPK phosphorylation in HCAECs treated with vehicle, CRP, JNK inhibitor (SP600125), p38 MAPK inhibitor (SB203580), JNK inhibitor with CRP or p38 MAPK with CRP. (B) RAGE expression in HCAECs. Data are represented as the mean $\pm \operatorname{SEM}(n=3-6) .{ }^{\mathrm{b}} P<0.05,{ }^{\mathrm{c}} P<0.01$ vs vehicle. ${ }^{\mathrm{e}} P<0.05$ vs CRP.

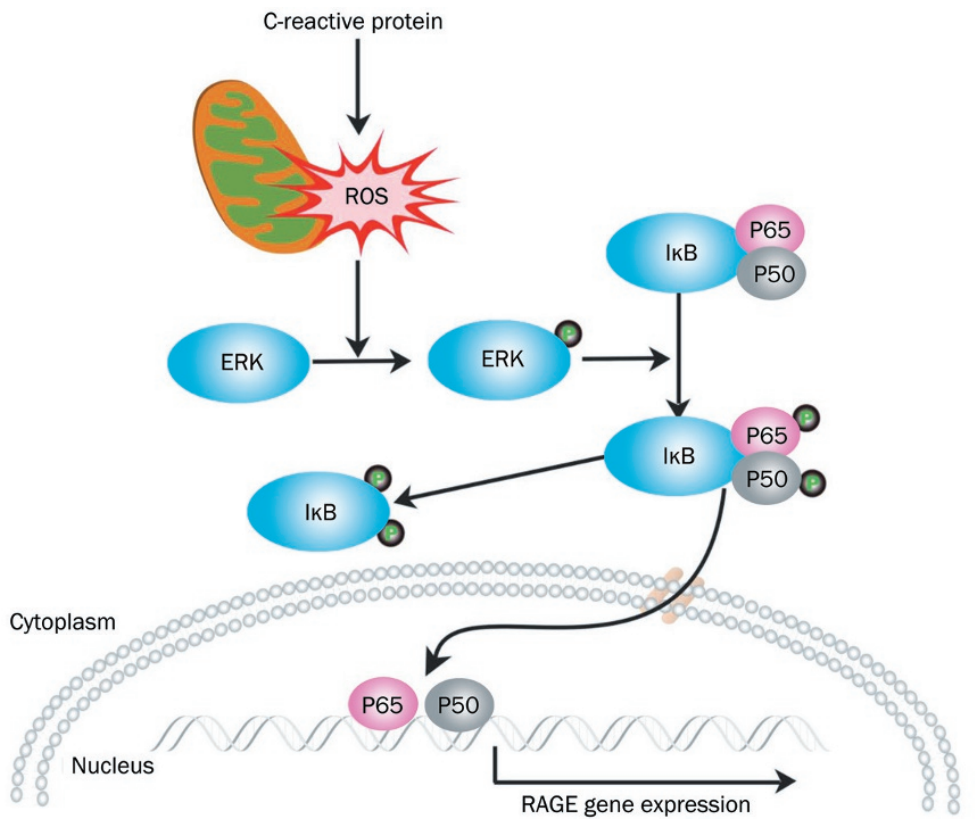

Figure 5. Schematic representation of the signaling cascades involved in CRP-induced RAGE expression in HCAECs. CRP stimulates mitochondrial ROS generation, ERK activation and NF-KB subunit phosphorylation and nuclear translocation leading to RAGE expression. 
therefore, provides a novel target for the prevention and treatment of vascular diseases.

\section{Acknowledgements}

This work was supported by Guangdong Natural Science Foundation (No S2011020002143), National Natural Science Foundation of China (NSFC, No 30971253) and the Ministry of Education of the People's Republic of China (Doctoral Program Research Fund, 20094423110001).

\section{Author contribution}

Yun ZHONG, Chuan-fang CHENG, and Shi-ming LIU designed the study; Yun ZHONG, Chuan-fang CHENG, Yizhi LUO, Chao-wei TIAN, Hui YANG, and Ben-rong LIU conducted the experiment; Yun ZHONG, Chuan-fang CHENG, Min-sheng CHEN, Yan-fang CHEN, and Shi-ming LIU analyzed the data; and Yun ZHONG, Chuan-fang CHENG, Yanfang CHEN, and Shi-ming LIU wrote the paper.

\section{References}

1 Ridker PM. C-reactive protein and the prediction of cardiovascular events among those at intermediate risk: Moving an inflammatory hypothesis toward consensus. J Am Coll Cardiol 2007; 49: 2129-38.

2 Bisoendial RJ, Boekholdt SM, Vergeer M, Stroes ES, Kastelein JJ. C-reactive protein is a mediator of cardiovascular disease. Eur Heart J 2010; 31: 2087-91.

3 Grad E, Danenberg HD. C-reactive protein and atherothrombosis: Cause or effect? Blood Rev 2013; 27: 23-9.

4 Sundgren NC, Zhu W, Yuhanna IS, Chambliss KL, Ahmed M, Tanigaki $\mathrm{K}$, et al. Coupling of Fcy receptor I to Fcy receptor Ilb by src kinase mediates c-reactive protein impairment of endothelial function. Circ Res 2011; 109: 1132-40.

5 Raaz-Schrauder D, Ekici AB, Klinghammer L, Stumpf C, Achenbach S, Herrmann M, et al. The proinflammatory effect of c-reactive protein on human endothelial cells depends on the Fcy lla genotype. Thromb Res 2014; 133: 426-32.

6 Libby P, Ridker PM, Hansson GK. Progress and challenges in translating the biology of atherosclerosis. Nature 2011; 473: $317-$ 25.

7 Tinkel J, Hassanain H, Khouri SJ. Cardiovascular antioxidant therapy: A review of supplements, pharmacotherapies, and mechanisms. Cardiol Rev 2012; 20: 77-83.

8 Munzel T, Gori T, Bruno RM, Taddei S. Is oxidative stress a therapeutic target in cardiovascular disease? Eur Heart J 2010; 31: 2741-8.

9 Zhang Z, Yang Y, Hill MA, Wu J. Does c-reactive protein contribute to atherothrombosis via oxidant-mediated release of pro-thrombotic factors and activation of platelets? Front Physiol 2012; 3: 433.

10 Singh U, Devaraj S, Jialal I. C-reactive protein decreases tissue plasminogen activator activity in human aortic endothelial cells: Evidence that c-reactive protein is a procoagulant. Arterioscler Thromb Vasc Biol 2005; 25: 2216-21.

11 Huang X, Zhang J, Liu J, Sun L, Zhao H, Lu Y, et al. C-reactive protein promotes adhesion of monocytes to endothelial cells via nadph oxidase-mediated oxidative stress. J Cell Biochem 2012; 113: 857-67.

12 Chen Y, Wang J, Yao Y, Yuan W, Kong M, Lin Y, et al. Crp regulates the expression and activity of tissue factor as well as tissue factor pathway inhibitor via NF-kappaB and ERK 1/2 MAPK pathway. FEBS Lett 2009; 583: 2811-8.

13 Mahajan N, Dhawan V. Receptor for advanced glycation end products (rage) in vascular and inflammatory diseases. Int J Cardiol 2013; 168 : 1788-94.
14 Morris-Rosenfeld S, Blessing E, Preusch MR, Albrecht C, Bierhaus $\mathrm{A}$, Andrassy $\mathrm{M}$, et al. Deletion of bone marrow-derived receptor for advanced glycation end products inhibits atherosclerotic plaque progression. Eur J Clin Investig 2011; 41: 1164-71.

15 Watson AM, Li J, Samijono D, Bierhaus A, Thomas MC, JandeleitDahm KA, et al. Quinapril treatment abolishes diabetes-associated atherosclerosis in rage/apolipoprotein $\mathrm{E}$ double knockout mice. Atherosclerosis 2014; 235: 444-8.

16 Mahajan N, Bahl A, Dhawan V. C-reactive protein (crp) up-regulates expression of receptor for advanced glycation end products (RAGE) and its inflammatory ligand EN-RAGE in THP-1 cells: Inhibitory effects of atorvastatin. Int J Cardiol 2010; 142: 273-8.

17 Zhong Y, Li SH, Liu SM, Szmitko PE, He XQ, Fedak PW, et al. C-reactive protein upregulates receptor for advanced glycation end products expression in human endothelial cells. Hypertension 2006; 48: 50411.

18 Wynants M, Vengethasamy L, Ronisz A, Meyns B, Delcroix M, Quarck R. Nf-kappab pathway is involved in CRP-induced effects on pulmonary arterial endothelial cells in chronic thromboembolic pulmonary hypertension. Am J Physiol Lung Cell Mol Physiol 2013; 305: L93442.

19 Devaraj S, Kumaresan PR, Jialal I. C-reactive protein induces release of both endothelial microparticles and circulating endothelial cells in vitro and in vivo: Further evidence of endothelial dysfunction. Clin Chem 2011; 57: $1757-61$.

20 Barlovic DP, Soro-Paavonen A, Jandeleit-Dahm KA. Rage biology, atherosclerosis and diabetes. Clin Sci (Lond) 2011; 121: 43-55.

21 Fujii H, Li SH, Szmitko PE, Fedak PW, Verma S. C-reactive protein alters antioxidant defenses and promotes apoptosis in endothelial progenitor cells. Arterioscler Thromb Vasc Biol 2006; 26: 2476-82.

22 Qamirani E, Ren Y, Kuo L, Hein TW. C-reactive protein inhibits endothelium-dependent no-mediated dilation in coronary arterioles by activating p38 kinase and nad(p)h oxidase. Arterioscler Thromb Vasc Biol 2005; 25: 995-1001.

23 Zhao XQ, Zhang MW, Wang F, Zhao YX, Li JJ, Wang XP, et al. Crp enhances soluble lox-1 release from macrophages by activating TNFalpha converting enzyme. J Lipid Res 2011; 52: 923-33.

24 Bello G, Cailotto F, Hanriot D, Kolopp-Sarda MN, Latger-Cannard V, Hess K, et al. C-reactive protein (crp) increases VEGF-A expression in monocytic cells via a PI3-kinase and ERK 1/2 signaling dependent pathway. Atherosclerosis 2008; 200: 286-93.

25 Cui C, Shi Q, Zhang X, Liu X, Bai Y, Li J, et al. Crp promotes MMP-10 expression via c-Raf/MEK/ERK and JAK1/ERK pathways in cardiomyocytes. Cell Signal 2012; 24: 810-8.

26 Pamukcu B, Lip GY, Shantsila E. The nuclear factor-kappa B pathway in atherosclerosis: a potential therapeutic target for atherothrombotic vascular disease. Thromb Res 2011; 128: 117-23.

27 Baker RG, Hayden MS, Ghosh S. NF-kappaB, inflammation, and metabolic disease. Cell Metab 2011; 13: 11-22.

28 Sun X, Icli B, Wara AK, Belkin N, He S, Kobzik L, et al. Microrna-181b regulates NF-kappaB-mediated vascular inflammation. J Clin Invest 2012; 122: 1973-90.

29 Liang YJ, Shyu KG, Wang BW, Lai LP. C-reactive protein activates the nuclear factor-kappab pathway and induces vascular cell adhesion molecule-1 expression through cd32 in human umbilical vein endothelial cells and aortic endothelial cells. J Mol Cell Cardiol 2006; 40: $412-20$.

30 Scirica BM, Morrow DA. Is c-reactive protein an innocent bystander or proatherogenic culprit? The verdict is still out. Circulation 2006; 113: 2128-34.

31 Vaishnav D, Jambal P, Reusch JE, Pugazhenthi S. Sp600125, an inhibitor of c-jun $n$-terminal kinase, activates CREB by a p38 MAPKmediated pathway. Biochem Biophys Res Commun 2003; 307: 85560. 